University of Massachusetts Amherst

ScholarWorks@UMass Amherst

Chemistry Department Faculty Publication Series

Chemistry

1987

\title{
Models for Dispersion in Flow Injection Analysis Part 1. Basic Requirements and Study of Factors Affecting Dispersion
}

David C. Stone

Loughborough University of Technology

Julian Tyson

University of Massachusetts Amherst

Follow this and additional works at: https://scholarworks.umass.edu/chem_faculty_pubs

Part of the Analytical Chemistry Commons

\section{Recommended Citation}

Stone, David C. and Tyson, Julian, "Models for Dispersion in Flow Injection Analysis Part 1. Basic Requirements and Study of Factors Affecting Dispersion" (1987). Analyst. 1397.

Retrieved from https://scholarworks.umass.edu/chem_faculty_pubs/1397 


\title{
Models for Dispersion in Flow Injection Analysis
}

\section{Part 1. Basic Requirements and Study of Factors Affecting Dispersion*}

\author{
David C. Stone and Julian F. Tyson \\ Department of Chemistry, University of Technology, Loughborough, Leicestershire LE11 3TU, UK
}

\begin{abstract}
The various approaches adopted for accounting for dispersion behaviour in flow injection analysis are examined and the advantages of a modelling approach are discussed. The variation of the dispersion coefficient as a function of (a) flow-rate, (b) tube length, (c) tube inner diameter and (d) method of injection obtained under typical flow injection conditions are studied and discussed. Explanations for some of the effects observed are presented in terms of molecular diffusion and convective flow patterns. The advantages of "time" injection over the more usual "slug" injection are clearly demonstrated and the use of the single well stirred tank model to describe the relationship between volume injected and dispersion coefficient under conditions of time injection are examined. The applications of this model to describing the entire curve shape for slug injection and of the potentially more versatile two-tank model are briefly introduced.
\end{abstract}

Keywords: Flow injection analysis; dispersion coefficient; flow models; time injection

Since the introduction of flow injection analysis (FIA) by Růžčka and Hansen, ${ }^{1}$ a number of approaches to the quantitative description of the dispersion processes that occur in FIA manifolds have been adopted. The starting point for such descriptions is the work of Taylor, ${ }^{2,3}$ who accounted for the dispersion of a solute flowing down a tube as the result of the combined effects of molecular diffusion and the parabolic velocity profile generated under conditions of laminar flow. The resulting concentration - time profile is given by the diffusion - convection equation, which may be expressed in the form

$$
\frac{\partial C}{\partial t}=D_{\mathrm{m}}\left(\frac{\partial^{2} C}{\partial x^{2}}+\frac{\partial^{2} C}{\partial r^{2}}+\frac{1}{r} \cdot \frac{\partial C}{\partial r}\right)-u_{\bullet}\left(1-\frac{r^{2}}{a^{2}}\right) \frac{\partial C}{\partial x}
$$

where $C$ is the concentration at the point $(x, r, t), D_{\mathrm{m}}$ the molecular diffusion coefficient, $x$ the distance along the tube axis, $r$ the radial distance from the tube centre, $t$ the point in time, $a$ the tube radius and $u_{\mathrm{o}}$ the linear flow velocity at the tube centre. Because equation (1) could not be solved directly, Taylor derived approximate solutions for two extreme cases: those of pure laminar flow in the absence of diffusion, and diffusion-controlled dispersion. The latter is the case where the contribution from laminar flow is effectively masked by the effects of molecular diffusion.

However, the conditions for which Taylor's solutions are valid lie outside the range of conditions normally encountered in FIA. Therefore, in order to describe dispersion quantitatively, either the diffusion - convection equation must be solved using a numerical method, or an appropriate flow model must be used.

The use of numerical integration procedures has been described by various workers ${ }^{4-7}$ for the prediction of sample bolus shapes both with and without a chemical reaction occurring. The main disadvantage of using such methods for solving the diffusion - convection equation is that they are applicable only to the use of a single-line manifold, and assume that the flow remains undisturbed by the valve, the injection process, the detector or any connections in the manifold. In practice, such conditions are hard to obtain, whilst many manifolds are much more complicated than the single-line case.

* Presented at SAC 86, the 7th SAC International Conference on Analytical Chemistry, Bristol, UK, 20-26 July, 1986.
The two most widely used flow models are the tanks-inseries and diffusion (axially dispersed plug flow) models, both of which were originally described in the chemical engineering literature. ${ }^{8}$ These models were used by Růžička and Hansen to discuss dispersion in simple, single-line manifolds. 9,10 They have also been used by numerous workers to discuss dispersion in coiled, packed-bed and single-bead string reactors, and to assess the relative performance of such reactors. ${ }^{11-15}$ One disadvantage of these models is that they are derived for tracer input conditions, i.e., the injection of unit concentration of solute in zero time (the so-called "delta" function). Clearly, such conditions are not met in practical FIA manifolds.

Recently, Gisin et al.16 have discussed the precision of gradient techniques in FIA. The hydrodynamically limited precision of concentration gradients produced by mixing tanks and straight capillary tubes was examined theoretically using four simple flow models. For mixing tanks, an exponential residence time distribution was convoluted with $(a)$ a plug injection function and $(b)$ an exponential wash-out function. For straight capillary tubes, the tanks-in-series model was convoluted with $(a)$ a plug injection function and $(b)$ a delta injection function. The resulting concentration gradients were expressed as a function of reduced time and reduced injection volume.

An alternative approach to the use of numerical techniques or flow models for the quantitative description of dispersion is the empirical method of Gomez-Nieto et al. ${ }^{17}$ This method uses multiple regression analysis on experimentally determined data to derive expressions for travel time, base-line width, time to peak maximum and dispersion coefficient for a single-line manifold. The main disadvantages of this method are the need for a large number of experimental measurements and the fact that the equations derived are applicable only to the specific conditions and manifold components employed.

For these reasons, various new models for dispersion are being examined 18 in the light of certain criteria. Firstly, such models should use relatively simple mathematical equations, and should be capable of predicting peak height, base-line width and peak shape, as such parameters are important in the design of manifolds for specific applications. Secondly, they should apply to a wide range of conditions, and should account for all the relevant flow injection variables.

In order to develop and evaluate such models, an extensive investigation of the factors affecting physical dispersion has been undertaken for a single-line manifold. Preliminary 
experiments on the effect of confluencing in more complex manifolds are in progress, but are not described in this paper. The results of studies on the effect of the solute used and the contribution of the flow cell used with spectrophotometric detection have been presented elsewhere. 19,20 In this paper, the results of studies on the effects of flow-rate, tube length and inner diameter and the method of injection are presented, together with some preliminary results for the use of flow models based on (a) a single well stirred mixing tank and (b) two well stirred tanks in series. A detailed study of the application of this second model will be presented in a later publication.

\section{Experimental}

A single-line manifold was used throughout. This was constructed from PTFE tubing of various inner diameters (RS Components, Anachem), a Rheodyne 5020 injection valve and a Gilson Minipuls-2 peristaltic pump. All tubing was kept as straight as possible. Long tubes were loosely coiled for convenience. A minimum coil diameter of $5 \mathrm{~cm}$ was employed, it having been established in separate experiments that a coil to tube diameter ratio of less than 15 was necessary before any significant effects due to coiling could be observed under the conditions used in these experiments. Sample solutions were tartrazine (Pointing Ltd., 0.25 and $0.020 \mathrm{~g} \mathrm{l}^{-1}$ ) and potassium permanganate (BDH Chemicals, analyticalreagent grade, 1.00 and $1.33 \mathrm{~g}^{-1}$ ). All solutions were prepared in distilled water, which was also used as the carrier stream. Results were recorded using either a Pye Unicam SP6-250 visible spectrophotometer with a W + W Tarkan 600 chart recorder, or a Pye Unicam PU8610 UV - visible spectrophotometer with a Philips PM8251 chart recorder. The flow cells used were an 8- $\mu$ l quartz cell (Pye Unicam) and a glass flow cell constructed in-house 19 to have an optical volume of approximately $0.6 \mu \mathrm{l}$. A Pye Unicam SP9 atomic absorption spectrometer was also used with an SP9 computer and a chart recorder.

\section{Effect of Flow-rate}

The dispersion coefficient, $D$, defined as the ratio of injected to peak concentrations, was measured as a function of flow-rate for $30-, 110-$ and $360-\mathrm{cm}$ lengths of $0.58 \mathrm{~mm}$ i.d. tubing using the $0.6-\mu \mathrm{l}$ flow cell. Ten replicate injections of 113 $\mu l$ (the minimum volume obtainable with the valve supplied) of a $0.25 \mathrm{~g} \mathrm{l}^{-1}$ tartrazine solution were made for each tube length at various flow-rates over the range $0.1-9.0 \mathrm{ml} \mathrm{min}^{-1}$. Each set of injections was followed by a measurement of the steady-state absorbance. The mean, standard deviation and $95 \%$ confidence interval about the mean were calculated for each measurement of the dispersion coefficient. Individual flow-rates were measured by collecting the effluent from the flow cell over a timed period and weighing.

\section{Effect of Tube Length}

The dispersion coefficient was measured for different lengths of $0.58 \mathrm{~mm}$ i.d. tubing over the range $19.3-350 \mathrm{~cm}$ using both solution spectrophotometric and flame AAS detection. Potassium permanganate was used as the sample because it can be monitored directly using both methods of detection. Similar sample concentrations and identical conditions were used for both methods in order to provide some comparison between the two. For flame AAS detection, manganese was monitored at $403.1 \mathrm{~nm}$ using an air - acetylene flame. The manifold tubing was connected directly to the nebuliser of the AAS instrument. A flow-rate of $5.8 \mathrm{ml} \mathrm{min}^{-1}$ was used throughout, which corresponded to the natural aspiration rate of the nebuliser under the conditions used. For solution spectrophotometric detection, the $0.6-\mu$ l flow cell was used, the absorbance of permanganate ion being monitored at $526 \mathrm{~nm}$. As the cell has such a low volume, the dispersion observed very closely approximates that due to the manifold, allowing the additional dispersion introduced by the nebuliser - spray chamber of the atomic absorption spectrometer to be evaluated.

Values of the dispersion coefficient were calculated from the mean peak heights of five replicate injections of $67.3 \mu \mathrm{l}$ of sample, the peak heights being first converted into concentration values by means of a calibration graph. The sample volume was reduced by shortening the connecting tubes of the sample loop to ensure that a dispersion coefficient greater than unity was obtained for short tube lengths. Because the breaking and making of connections introduces some uncertainty into the value of $D$ obtained (see later), each determination was carried out in triplicate, a connection being broken and re-made each time, and the mean, standard deviation and $95 \%$ confidence interval about the mean were calculated.

\section{Effect of Tube Inner Diameter}

The dispersion coefficient was measured at different flowrates over the range $0.5-6.0 \mathrm{ml} \mathrm{min}^{-1}$ for tube inner diameters of $0.3,0.5,0.8$ and $1.0 \mathrm{~mm}$. These internal diameters were chosen as they could all be supplied to a uniform specification by one manufacturer. The tube length was varied with the inner diameter in order to keep the tube volume constant. The tube volumes used were 106, 215 and $410 \mu \mathrm{l}$. The $0.6-\mu \mathrm{l}$ flow cell was used, the absorbance of tartrazine being monitored at $426 \mathrm{~nm}$. The inner diameter of the sample loop was not matched to that of the remainder of the manifold in order to maintain accurately a constant sample volume. Ten replicate injections of $75.9 \mu \mathrm{l}$ (replacement valve) of a solution containing $0.25 \mathrm{~g}^{-1}$ of tartrazine were made for each determination of the value of $D$, the mean, standard deviation and relative standard deviation being calculated. Peak shapes were also recorded using a fast chart speed.

\section{Method of Injection}

The injection valve was automated by means of a stepper motor and reduction gear drive (McLennan Servo Supplies), controlled by a dedicated microprocessor unit. This could be programmed to give any desired switching sequence, with a timing accuracy of $0.1 \mathrm{~ms}$ and precision for replicate injections typically better than $1 \%$ RSD. The valve was fitted with a $600-\mu l$ loop and connected to the $8-\mu l$ flow cell via $50 \mathrm{~cm}$ of $0.58 \mathrm{~mm}$ i.d. tubing. By varying the delay time between switching and returning the valve, different injection volumes were obtained. The volume injected was calculated from the volume flow-rate and the delay time, by assuming that the valve had a negligible residual volume.

Peak shapes were recorded for injection volumes over the range $10-600 \mu \mathrm{l}$, using a flow-rate of $1.23 \mathrm{ml} \mathrm{min}^{-1}$ and a fast chart speed. The dispersion coefficient was measured for sample volumes over the range $3.2-315 \mu \mathrm{l}$ using a flow-rate of $1.89 \mathrm{ml} \mathrm{min}^{-1}$. The range of sample volumes was chosen so that no diluted sample entered the manifold from the sample loop (see later). Ten replicate injections were made for each measurement, and the mean, standard deviation and $95 \%$ confidence interval about the mean were calculated.

\section{Results and Discussion}

\section{Effect of Flow-rate}

The results obtained for the variation of the dispersion coefficient with flow-rate are shown in Fig. 1. With the apparatus used, relative standard deviations for ten replicate injections were typically less than $1 \%$, but poorer precision was found for the value of $D$ obtained under identical conditions on a day-to-day basis. For example, for a tube 


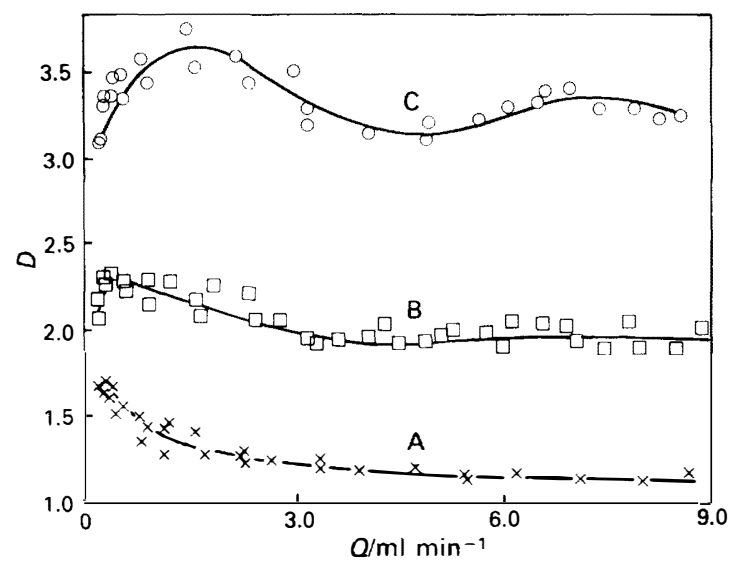

Fig. 1. Variation of $D$ with flow-rate for different tube lengths: (A) $L=30 \mathrm{~cm}$; (B) $110 \mathrm{~cm}$; and (C) $360 \mathrm{~cm}$

length of $110 \mathrm{~cm}$, a sample volume of $113 \mu \mathrm{l}$ and a flow-rate of $5.00 \mathrm{ml} \mathrm{min}^{-1}$, the mean of ten consecutive determinations of $D$ was found to be 1.95 with a $95 \%$ confidence interval of $\pm 0.012( \pm 0.6 \%$ of the mean value). However, when the same measurements were performed over several days with the breaking and making of connections, the value of $D$ obtained was 1.90 with a $95 \%$ confidence interval $\pm 0.089( \pm 5 \%$ of the mean value).

This made it difficult to define accurately the way in which the value of $D$ varied with flow-rate for the different tube lengths without considerable additional and time-consuming work. However, comparison of the confidence intervals for individual points for any given tube length revealed that the observed changes in the value of $D$ with flow-rate represented a real effect.

For short tube lengths, the value of $D$ was found to decrease with increasing flow-rate, becoming constant above a flowrate of approximately $5 \mathrm{ml} \mathrm{min}-1$. As the tube length was increased, the value of $D$ passed through a maximum at low flow-rates, again becoming constant above a flow-rate of about $5 \mathrm{ml} \mathrm{min}^{-1}$. For long tube lengths, two maxima were observed, one at about $1.5 \mathrm{ml} \mathrm{min}^{-1}$ and the other at about 6.5 $\mathrm{ml} \mathrm{min}^{-1}$.

Results obtained for different sample volumes and tube lengths confirmed the results obtained, the values of $D$ increasing with decreasing sample volume, as would be expected. For example, the results obtained for a sample volume of $67.3 \mu \mathrm{l}$ and a tube length of $30 \mathrm{~cm}$ were similar to those obtained for a sample volume of $113 \mu \mathrm{l}$ and a tube length of $70 \mathrm{~cm}$.

The observed variation in the dispersion coefficient with flow-rate for the different tube lengths reflects a variation in the relative contributions of the convection and diffusion mechanisms to the over-all dispersion of the sample zone. It is well established that the convection process (distortion due to laminar flow) will dominate for short tube lengths and high flow-rates, whereas the diffusion process will dominate for long tubes and low-flow rates. ${ }^{8}$ Using Taylor's equations for dispersion by convection alone, ${ }^{2}$ it can be shown that for the introduction of a volume $V_{\mathrm{s}}$ of sample of unit concentration into a length of tubing of volume $V_{\mathrm{R}}$, at a volume flow-rate $Q$, the concentration - time distribution at the tube outlet will be given by

$$
\begin{array}{lllll}
\mathrm{t} \leqslant t_{\mathrm{o}} & C=0 \quad \ldots \quad \ldots & \ldots & \ldots \\
t_{\mathrm{o}}<t<t_{\mathrm{p}} & C=1-\left(V_{\mathrm{R}} / 2 Q t\right) & \ldots & \ldots \\
t=t_{\mathrm{p}} & C_{\mathrm{p}}=V_{\mathrm{s}} /\left(V_{\mathrm{s}}+V_{\mathrm{R}}\right) & \ldots & \ldots \\
t>t_{\mathrm{p}} & C=V_{\mathrm{s}} / 2 Q t & \ldots & \ldots & \ldots
\end{array}
$$

where $t_{\mathrm{o}}$ is the appearance time $\left(=V_{\mathrm{R}} / 2 Q\right), t_{\mathrm{p}}$ is the time to the

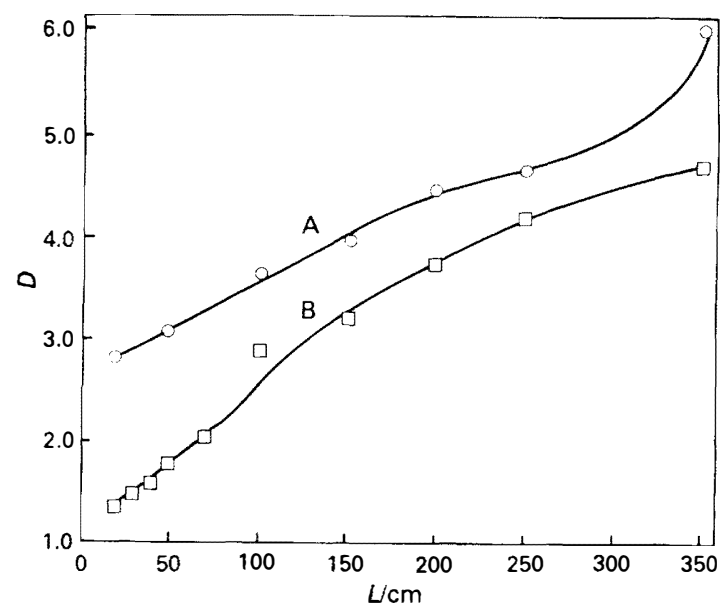

Fig. 2. Variation of $D$ with tube length for (A) flame AAS and (B) solution spectrophotometric detection

peak maximum $\left[=\left(V_{\mathrm{R}}+V_{\mathrm{s}}\right) / 2 Q\right]$ and $C_{\mathrm{p}}$ is the peak concentration. Equation (2c) predicts that, for dispersion by convection alone, the value of $D\left(=1 / C_{\mathrm{p}}\right.$ for a sample of unit concentration) will be independent of flow-rate. Thus the results obtained for a tube length of $30 \mathrm{~cm}$ may be interpreted as representing the situation where dispersion occurs predominantly by convection, with an increasing contribution from molecular diffusion at lower flow-rates. As the residence time of the sample zone in the manifold is increased (longer tube length, lower flow-rate), there will be an increasing contribution from molecular diffusion to the dispersion of the sample zone. This is reflected in the increasing complexity of the dispersion coefficient - flow-rate relationships for increasing tube length, although a full explanation for the shape of these curves has not yet been found.

\section{Effect of Tube Length}

The results obtained for the variation of the dispersion coefficient with tube length for the two different methods of detection are shown in Fig. 2. These results highlight the effect of the nebuliser - spray chamber of the atomic absorption spectrometer on the observed sample dispersion. Graph A shows the results obtained using the atomic absorption spectrometer and graph B shows those obtained using the solution spectrophotometer. Comparison of the two shows that for short tube lengths, the nebuliser - spray chamber assembly makes a large contribution to the observed sample dispersion, the value of $D$ obtained for a tube length of 19.3 $\mathrm{cm}$ being double that produced by the manifold alone. This effect appears to become less significant as the tube length is increased.

Both graphs show a linear portion up to a tube length of about $100 \mathrm{~cm}$. For graph $\mathrm{A}$, linear regression analysis on the first three points gave a correlation coefficient of 0.9970 with slope 0.0103 and intercept 2.60 . For graph $B$, for the first five points the correlation coefficient was 0.9986 with slope 0.0138 and intercept 1.07. Putting $V_{\mathrm{R}}=\pi d^{2} L / 4$, where $d$ is the tube inner diameter and $L$ the length, into equation (2c) gives

$$
C_{\mathrm{p}}=V_{\mathrm{s}} /\left[V_{\mathrm{s}}+\left(\pi d^{2} L / 4\right)\right] \quad \ldots \quad \ldots
$$

Putting $D=1 / C_{\mathrm{p}}$ and rearranging equation (3) gives

$$
D=1+\pi d^{2} L / 4 V_{\mathrm{s}} \quad \ldots \quad \ldots
$$

This predicts that, for dispersion by convection alone, there is a linear relationship between the dispersion coefficient and tube length, with slope $\pi d^{2} / 4 V_{\mathrm{s}}$ and intercept 1 . Hence the results obtained for tube lengths of less than $100 \mathrm{~cm}$ suggest 
that, for the flow-rate used, dispersion occurs predominantly by convection. This is confirmed by comparison with Fig. 1, which suggests that this should indeed be so. However, neither the intercept nor the slope give the values expected on the basis of equation (4), indicating that dispersion is not occuring purely by convection.

Similar results to graph B have been obtained for different experimental conditions, the particular shape depending on tube length and flow-rate.

\section{Effect of Tube Inner Diameter}

Some results obtained for the variation of the dispersion coefficient with flow-rate for different tube diameters and constant tube volume are shown in Fig. 3. It was found that very few results could be obtained for tubing of $0.3 \mathrm{~mm}$ i.d. with the apparatus used, because of the high back-pressure generated by such tubing. The results obtained using the other tube diameters $(0.5,0.8$ and $1.0 \mathrm{~mm})$ show that the effect on the dispersion coefficient of changing the tube diameter for a constant tube volume is dependent on flow-rate. In general, the value of $D$ was found to increase with increasing tube diameter, although for certain combinations of tube volume and flow-rate the reverse was true, whereas in other instances no clear trend emerged.

Considerable variation was found in peak shape between the different diameters of tubing. Representative examples of the different peak shapes observed are shown in Fig. 4. These will be referred to as $(a)$ skewed Gaussian, (b) triangular, (c) humped, $(d)$ tailed and $(e)$ exponential. Such peak shapes can be explained in terms of the mixing patterns existing within the flow manifold, following chemical engineering practice. ${ }^{21}$

Skewed Gaussian peaks were obtained for $0.5 \mathrm{~mm}$ i.d. tubing for all the flow-rates and tube volumes examined, and are assumed to arise from pure diffusion - convection mechanisms. Humped peaks were obtained for both 0.8 and $1.0 \mathrm{~mm}$ i.d. tubing, and can be explained by the process of "channelling." When two flow paths exist through a reactor or manifold, one being longer than the other, the result will be two overlapping concentration - time graphs, giving a humped or, in extreme circumstances, a double peak (Fig. 5). Triangular peak shapes were observed only for $0.8 \mathrm{~mm}$ i.d. tubing, and are probably an intermediate form of the skewed Gaussian and humped peak shapes.

Tailed peaks will result if there is a significant "dead volume" in the flow manifold. This is an extreme form of channelling, where solute is exchanged between regions of flowing and regions of stagnant water, resulting in a prolonged washout of the solute from the manifold. Such dead volumes are likely to occur wherever there are abrupt changes of bore or sharp bends in the flow manifold, such as in the flow cell, connections or injection valve. Tailed peaks were observed only for $1.0 \mathrm{~mm}$ tubing.

Exponential peaks will arise when the primary (diffusion convection) and secondary (channelling, dead volume) mixing processes combine so that the flow manifold behaves as if it were a small, well stirred mixing tank. Exponential peak shapes were observed for both 0.8 and $1.0 \mathrm{~mm}$ i.d. tubing, and have also been reported by other workers for short, fat tubes. 22,23

In all these experiments the tube diameter was varied whilst keeping the tube volume constant. However, if the tube diameter is varied whilst keeping the tube length constant, the dispersing volume will increase with increasing tube diameter, as $V=\pi d^{2} L / 4$. Therefore, one would expect the dispersion coefficient to increase with increasing tube diameter under these conditions. In a separate experiment, when the tube diameter was varied for a constant tube length, the value of $D$ was found to increase with increasing tube diameter as expected.

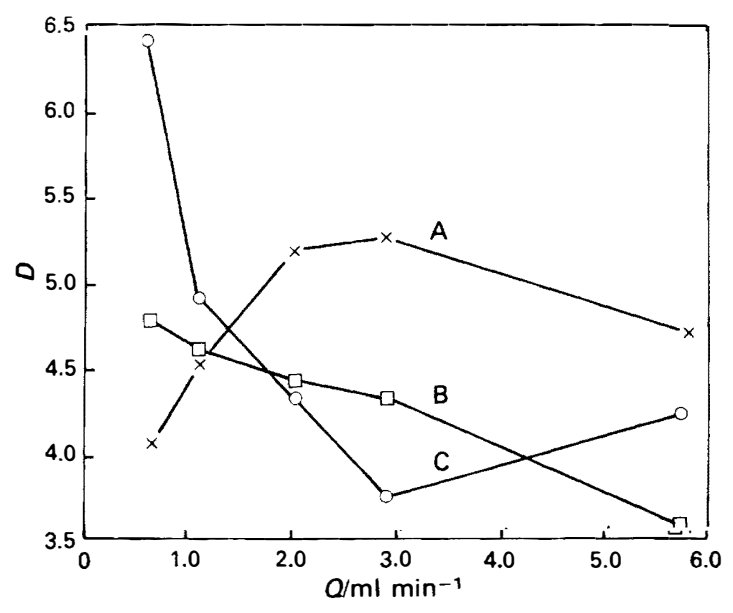

Fig. 3. Variation of $D$ with flow-rate for different tube diameters for a tube volume of $410 \mu \mathrm{l} . d=(A) 0.5 \mathrm{~mm}$; (B) $0.8 \mathrm{~mm}$; and (C) $1.0 \mathrm{~mm}$
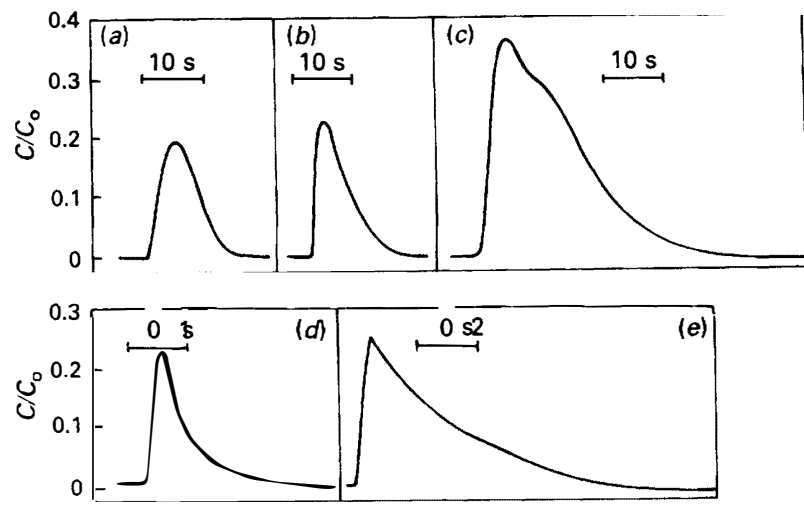

Fig. 4. Different types of peak shape obtained for different conditions. $V_{\mathrm{s}}=75.9 \mu \mathrm{l} . d=(a) 0.5 \mathrm{~mm} ;(b)$ and $(c) 0.8 \mathrm{~mm}$; and $(d)$ and $(e) 1.0 \mathrm{~mm} . Q=(a) 2.88 \mathrm{ml} \mathrm{min}^{-1}$; (b) $2.91 \mathrm{ml} \mathrm{min}^{-1}$; (c) 0.72 $\mathrm{ml} \mathrm{min}^{-1} ;(d) 2.90 \mathrm{ml} \mathrm{min}^{-1}$; and $(e) 0.55 \mathrm{ml} \mathrm{min}^{-1} . V_{\mathrm{T}}=(a),(b)$ and (d) $410 \mu \mathrm{l} ;(c) 106 \mu \mathrm{l}$; and (e) $215 \mu \mathrm{l}$

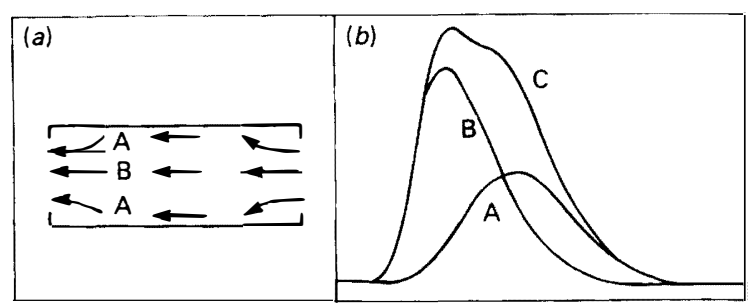

Fig. 5. Formation of a humped peak by channelling through a reactor. (a) Alternative flow paths; $(b)$ resulting peak shapes. Overlap of lines $A$ and $B$ produces line $C$

\section{Method of Injection}

The peak shapes obtained for different delay times, and hence different sample volumes, are shown in Fig. 6. Under the conditions used in this experiment, the steady state was achieved for sample volumes greater than $287 \mu \mathrm{l}$. For sample volumes over the range $10-410 \mu \mathrm{l}$, the fall curve was found to be the reverse of the rise curve to the steady state [Fig. $6(\mathrm{~A})-(\mathrm{F})]$. However, this did not occur when the full sample volume of $600 \mu \mathrm{l}$ was allowed to enter the manifold [Fig. 6 $(\mathrm{H})]$. The difference between these results can be explained by considering the mode of operation of the injection valve. 


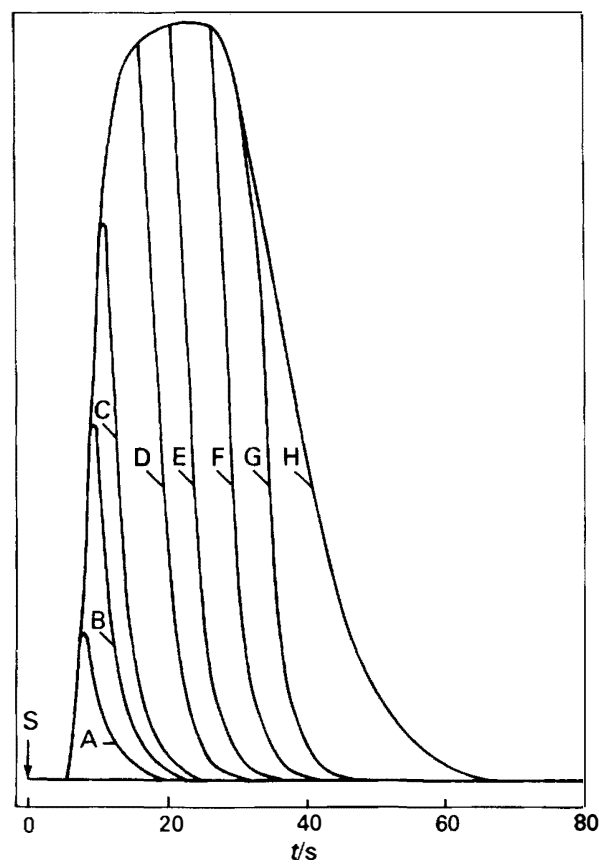

Fig. 6. Peak shapes obtained for different injection volumes: $V_{\mathrm{s}}=$ (A) $10 \mu \mathrm{l}$; (B) $41 \mu \mathrm{l}$; (C) $82 \mu \mathrm{l}$; (D); $205 \mu \mathrm{l}$; (E) $287 \mu \mathrm{l}$; (F) $410 \mu \mathrm{l}$; (G) $533 \mu \mathrm{l} ;(\mathrm{H}) 600 \mu \mathrm{l}$. S indicates the point of injection

(a)

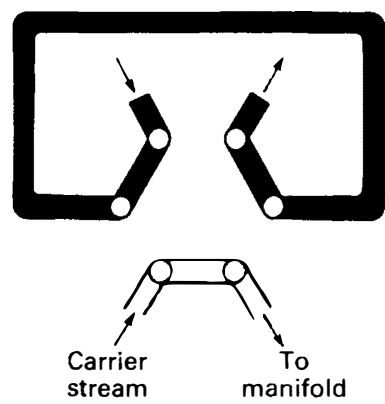

(b)

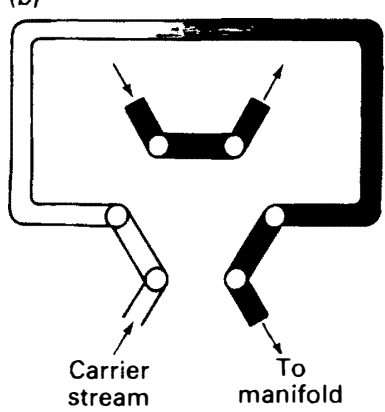

Fig. 7. Schematic diagram of the injection valve: (a) Loop fill position; $(b)$ injection position

Used in the conventional mode, the sample loop is first filled with sample [Fig. 7(a)]. When the sample is injected [Fig. 7(b)], the contents of the loop are flushed into the manifold by the carrier stream. This means that, whilst the front of the sample zone undergoes dispersion only in the manifold, the rear of the sample zone undergoes dispersion in both the manifold and the sample loop, i.e., the tail of the sample zone will become dispersed to a greater extent than the front. This mode of injection corresponds to that defined by

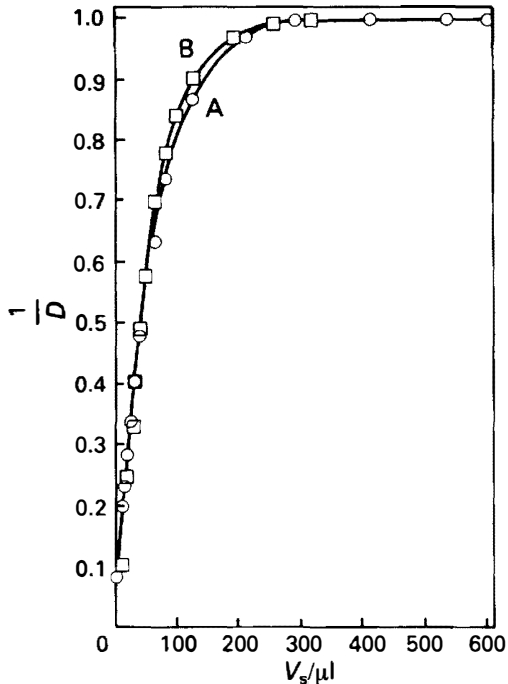

Fig. 8. Variation of $1 / D$ with sample volume. Flow-rate: (A) 1.23

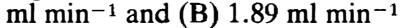

Reijn et al. ${ }^{24}$ as "slug" injection, in which a specific volume of the sample solution is instantaneously intercalated into the carrier stream.

If, however, the valve is switched back to the "fill" position before the dispersed tail of the sample zone can enter the manifold, then the rear of the sample zone in the manifold will become dispersed to the same extent as the front. This corresponds to "time" injection ${ }^{24}$ in which the sample solution is introduced for a specific period of time. Under these conditions, the fall curve is expected to be the reverse of the rise curve to the steady state, in contrast to slug injection, where this is not expected to be true. Hence Fig. 6(A)-(F) correspond to time injection, whereas Fig. $6(\mathrm{H})$ corresponds to slug injection. Fig. $6(\mathrm{G})$ shows the effect of switching back the valve after the dispersed rear of the sample zone has started to enter the manifold, the fall curve initially following that for slug injection and then reverting to that for time injection. It is expected that the differences between the two methods of injection will be most significant when the sample volume accounts for a large part of the total volume of the manifold (low dispersion systems), as for small sample volumes (high dispersion systems) any dispersion occurring in the sample loop will be negligible in comparison with that occurring in the remainder of the manifold. Similar considerations apply to the contributions from changes in channel geometry and bore within the valve and connecting tubing.

Values of the dispersion coefficient were calculated from the peaks shown in Fig. 6, and plotted together with the results obtained at a flow-rate of $1.89 \mathrm{ml} \mathrm{min}^{-1}$ as $1 / D$ against $V_{\mathrm{s}}$, the sample volume (Fig. 8). It was found that the value of $1 / D$ became constant for very low sample volumes, poor reproducibility being obtained for replicate injections. This indicates that the residual volume of the valve becomes increasingly significant at very low sample volumes. All these results were obtained using time injection.

The results obtained were tested for agreement with the relationship given by Růžička and Hansen 9,10 that

$$
\frac{1}{D}=1-\exp \left(-K V_{\mathrm{s}}\right) \quad \ldots \quad \ldots \quad \ldots \quad \ldots
$$

where $K=0.693 / V_{\frac{1}{2}}$ and $V_{\frac{1}{2}}$ is defined as the sample volume required to give a value of $D=2$, i.e, a dilution of two. Equation (5) is, in fact, the appropriate equation for the single 
stirred mixing tank model and should be compared with equation (7b) given later. Rearranging equation (5) gives

$$
-\operatorname{Ln}(1-1 / D)=K V_{\mathrm{s}} \quad \ldots \quad \ldots
$$

Therefore, if equation (5) is valid for the conditions employed in these experiments, a plot of $-\ln (1-1 / D)$ against $V_{\mathrm{s}}$ should be linear with a slope of $0.693 / V_{\frac{1}{2}}$.

The results obtained are shown in Fig. 9. For a flow-rate of $1.89 \mathrm{ml} \mathrm{min}^{-1}$, good agreement was found between the experimental data and equation (5) except for very low sample volumes. Linear regression analysis on the data gave a slope of 0.0186 with a correlation coefficient of 0.9996 , giving $V_{\frac{1}{2}}=37$ $\mu$ l. The value of $V_{\frac{1}{2}}$ obtained by interpolation from Fig. 8 was $40 \mu \mathrm{l}$. For a flow-rate of $1.23 \mathrm{ml} \mathrm{min}^{-1}$, equation (5) was found not to hold for sample volumes greater than $200 \mu \mathrm{l}$. Linear regression analysis on the data for sample volumes of less than $200 \mu$ l gave a slope of 0.0158 with a correlation coefficient of 0.9994 and $V_{\frac{1}{2}}=44 \mu \mathrm{l}$. This compares favourably with the value of $40 \mu \mathrm{l}$ obtained by interpolation from Fig. 8 . The breakdown of equation (5) for values of $D$ close to unity (peak heights close to the steady-state value) can be explained by consideration of the exponential term involving $K$ and $V_{\mathrm{s}}$. This predicts that the steady state $(D=1)$ will only be obtained for an infinite volume, whereas for the experimental conditions used the steady state could be achieved for sample volumes greater than 200 and $315 \mu \mathrm{l}$ for flow-rates of 1.23 and 1.89 $\mathrm{ml} \mathrm{min}^{-1}$, respectively. Therefore, it is predicted that equation (5) will always fail for sample volumes giving peak heights close or equal to the steady-state value. The fit of equation (5) to data generated using slug injection is currently being investigated. ${ }^{18}$

\section{Use of Simple Flow Models}

One of the simplest flow models for dispersion in FIA is the well stirred mixing tank model of Tyson and Idris. ${ }^{25}$ In this model, a slug of sample of volume $V_{\mathrm{s}}$ and initial concentration $C_{0}$ is allowed to flow into a well stirred mixing tank of volume $V_{\mathrm{m}}$. The resulting concentration - time profile at the tank outlet is then given by

$$
\begin{aligned}
& 0<t<t_{\mathrm{p}} \quad C=C_{0}\left[1-\exp \left(-Q t / V_{\mathrm{m}}\right)\right] \quad \ldots \\
& t=t_{\mathrm{p}} \quad C_{\mathrm{p}}=C_{0}\left[1-\exp \left(-V_{\mathrm{s}} / V_{\mathrm{m}}\right)\right] \quad \ldots \\
& t>t_{\mathrm{p}} \quad C=C_{\mathrm{p}} \exp \left[-Q\left(t-t_{\mathrm{p}}\right) / V_{\mathrm{m}}\right] \ldots
\end{aligned}
$$

where $t_{\mathrm{p}}=V_{\mathrm{s}} / Q$ and all other symbols are as defined earlier. This model has been applied successfully to FIA - AAS, ${ }^{25}$ and forms the basis of a number of peak width methods. ${ }^{26,27}$ Fig. 10(a) illustrates how the well stirred tank model (WSTM) compares with experimental data. As can be seen, the model fits portions of the rise and fall curves very well, but fails to predict the point of inflection on the initial rise curve, and shows a sharp discontinuity at the peak maximum not present in the experimental data.

Developments of the WSTM include the extended single tank and two tanks in parallel models used by Appleton and Tyson $^{28}$ to describe the behaviour of the nebuliser - spray chamber assembly of a flame atomic absorption spectrometer, and the two tanks-in-series (TTS) model. The last model assumes that the effluent from one well stirred tank immediately enters a second tank, and differs from the tanks-inseries model for $N=2$ in that it uses tanks of different volumes, and step changes in the input concentration rather than the delta function. The use of this model is illustrated in Fig. $10(b)$, and seems to offer greater flexibility than the WST model, which is really applicable only to manifolds producing exponential gradients, as far as predicting the entire peak shape is concerned. However, the WST model may have a limited applicability in predicting the effect of changing the injection volume, particularly if time injection is used.

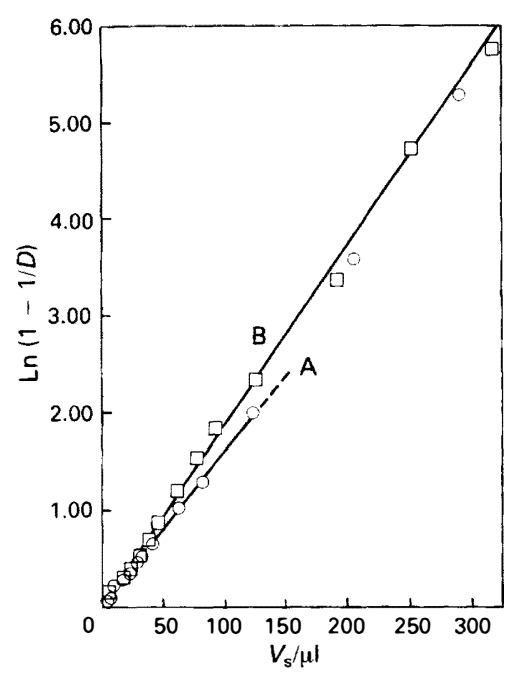

Fig. 9. Fit of the experimental data to equation (5). $Q=$ (A) 1.23 $\mathrm{ml} \mathrm{min-1}$ and (B) $1.89 \mathrm{ml} \mathrm{min}^{-1}$

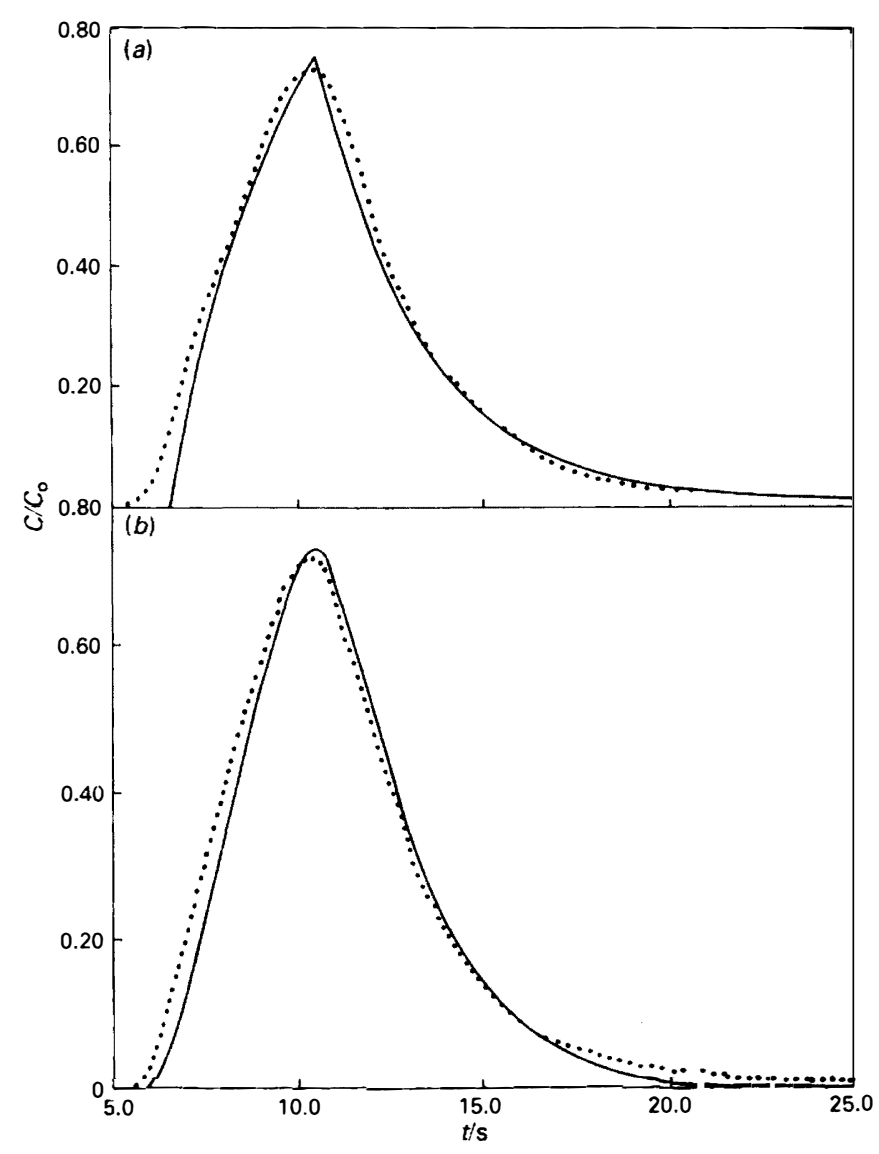

Fig. 10. Use of simple flow models. Experimental peak shape for $L=50 \mathrm{~cm}, d=0.58 \mathrm{~mm}, V_{\mathrm{s}}=82 \mu \mathrm{l}$ and $Q=1.23 \mathrm{ml} \mathrm{min}^{-1}$ (shown dotted). (a) WSTM with $V_{\mathrm{m}}=60 \mu \mathrm{l}$; and $(b)$ TTS model with $V_{1}=, 30$ $\mu \mathrm{l}, V_{2}=35 \mu \mathrm{l}$

\section{Comparison with Previous Results}

Růžička and Hansen ${ }^{9}$ have presented practical guidelines for designing flow injection manifolds. Of particular relevance to the work presented in this paper are their findings that $(a)$ dispersion increases with increasing sample volume according to equation $(5),(b)$ dispersion increases with the square root of the distance travelled, or with the square root of the residence time (equivalent to the time of appearance of the peak maximum), (c) dispersion increases with increasing 
flow-rate and $(d)$ the dispersion coefficient is independent of tube diameter for manifolds having the same residence time. it is therefore important to compare the findings of Růžčka and Hansen with the results presented in this paper.

The effect of sample volume on dispersion has already been discussed (see above). Results obtained using time injection are in agreement with those of Růžička and Hansen. ${ }^{9,10}$ However, preliminary results obtained using slug injection suggest that equation (5) breaks down for large sample volumes. Further investigation of this is currently in progress.

Before considering the effect of flow-rate, tube length and tube diameter, it should be pointed out that whereas Rủzička and Hansen used an $18-\mu$ l flow cell, a $0.6-\mu l$ flow cell was used in the work described here. Experience gained using different sizes and types of flow cell indicates that such a difference can have a profound influence on the observed dispersion behaviour of a flow injection manifold. ${ }^{19}$ Therefore, considerable caution must be exercised when comparing experimental results.

Růžička and Hansen reported a linear relationship between $C_{\mathrm{p}}$ and $L^{\frac{1}{2}}$. However, the results obtained were plotted as peak height and $D_{\mathrm{f}}$ (the dispersion coefficient "of the flow arrangement") against $t_{\mathrm{p}}^{\frac{1}{2}}$, making the assumption that $t_{\mathrm{p}}=\pi r^{2} / Q$, where $r$ is the tube radius [equation (16) in reference 9]. Assuming this approach to be valid, close examination of the experimental data presented (Fig. 15 in reference 9) reveals some deviation from the rule that $D=$ $K L^{\frac{1}{2}}$, where $K$ is an arbitrary constant [cf., equation (22) in reference 9]. This rule fails to satisfy the requirement that when $L=0, D=1$. Appleton and Tyson ${ }^{28}$ have proposed the expression

$$
(D-1)=k L^{\frac{1}{2}}
$$

When the data shown in Fig. 1 were plotted as $D$ against $L^{\frac{1}{2}}$, a straight line was obtained for tube lengths greater than 100 $\mathrm{cm}$, with a positive intercept greater than 1 , showing limited agreement with equation (8). This is in agreement with the results obtained by Appleton and Tyson. ${ }^{28}$

Examination of the results obtained by Rưžička and Hansen (Fig. 16 in reference 9) shows that dispersion increases with increasing flow-rate, in accordance with their rule 1. However, they only examined flow-rates of $0.25,0.75$ and 1.5 $\mathrm{ml} \mathrm{min-1}$. It should also be remembered that they employed a much larger flow cell than that used in the work described here. Results obtained using an $8-\mu$ l flow cell (not presented in this paper) show that dispersion increases with increasing flow-rate over the range $1-6 \mathrm{ml} \mathrm{min}^{-1}$ for tube lengths greater than $90 \mathrm{~cm}$, and is independent of flow-rate over the same range for tube lengths less than $90 \mathrm{~cm}$. This suggests that the differences between the results of Rưžička and Hansen and those presented here are due, to a large extent, to the different flow cells employed. These results also highlight the danger of deriving generalised expressions from limited experimental data.

Similar considerations apply to the results obtained for the effect of tube diameter. Thus Růžička and Hansen concluded that, "within the range of tube lengths and diameters used . . . $D_{\mathrm{f}}$ is independent of the tube diameter for the same residence time," whereas the results obtained in this work show a marked variation of the dispersion coefficient with tube diameter (Fig. 3). Růžička and Hansen did not examine the effect of tube diameter on peak shape.

\section{Conclusions}

It has been shown that physical dispersion is a complex function of many different factors, including flow-rate, tube length and diameter, method of injection and detector type. It has also been shown that although the primary dispersion mechanism is that of diffusion - convection, secondary dispersion mechanisms can also exist. This will especially be true for manifolds containing packed columns, tightly coiled tubes and gradient tanks. It therefore seems unlikely that a rigorous theoretical treatment of flow injection manifolds will be successful in producing accurate equations describing dispersion behaviour. On the other hand, a completely empirical approach would be time consuming and have no predictive power. Hence the use of suitable flow models to describe the dispersion processes would seem to be an attractive proposition. Such models must be able to account for those factors which affect dispersion, and must cover the range of conditions employed in FIA if they are to be of practical use. The preliminary results presented in this paper suggest that several flow models may be needed to meet these requirements.

The use of time injection has also been demonstrated, and would seem to offer a convenient means of increasing sample throughput whilst maintaining sensitivity by eliminating the contribution of the injection valve to peak broadening.

The authors thank Mr. B. Wilson of Pye Unicam for the use of the motor controller described in this paper. Financial support from the SERC and Pye Unicam is gratefully acknowledged.

\section{References}

1. Růžička, J., and Hansen, E. H., Anal. Chim. Acta, 1975, 78, 145.

2. Taylor, G., Proc. R. Soc. London, Ser. A, 1953, 219, 186

3. Taylor, G., Proc. R. Soc. London, Ser. A, 1954, 225, 473

4. Vanderslice, J. T., Stewart, K. K., Rosenfeld, A. G., and Higgs, D., Talanta, 1981, 28, 11

5, Vanderslice, J. T., Beecher, G. R., and Rosenfeld, A. G., Anal. Chem., 1984, 56, 292.

6. Painton, C. C., and Mottola, H. A., Anal. Chim. Acta, 1984, $158,67$.

7. Wada, H., Hiraoka, S., Yuchi, A., and Nakagawa, G., Anal. Chim. Acta, 1986, 179, 181.

8. Levenspiel, O., "Chemical Reaction Engineering," Second Edition, Wiley, New York, 1972, Chapter 9.

9. Růžička, J., and Hansen, E. H., Anal. Chim. Acta, 1978, 99, 37.

10. Ramsing, A. U., Růžička, J., and Hansen, E. H., Anal. Chim. Acta , 1981, 129, 1.

11. Tijssen, R., Anal. Chim. Acta, 1980, 114, 71.

12. Van Den Berg, J. H. M., Deelder, R. S., and Egberink, H. G. M., Anal. Chim. Acta, 1980, 114, 91 .

13. Reijn, J. M., Van Der Linden, W. E., and Poppe, H., Anal. Chim. Acta, 1981, 126, 1 .

14. Reijn, J. M., and Poppe, H., Anal. Chim. Acta, 1983, 145, 59.

15. Reijn, J. M., Poppe, H., and Van Der Linden, W. E., Anal. Chem., 1984, 56, 943.

16. Gisin, M., Thommen, C., and Mansfield, K. F., Anal. Chim. Acta, 1986, 179, 149.

17. Gomez-Nieto, M. A., Luque de Castro, M. D., Martin, A., and Valcarcel, M., Talanta, 1985, 32, 319.

18. Stone, D. C., and Tyson, J. F., work in progress.

19. Stone, D. C., and Tyson, J. F., Anal. Proc., 1986, 23, 23.

20. Stone, D. C., and Tyson, J. F., Anal. Chim. Acta, 1986, J/9, 427.

21. Wen, C. Y., and Fan, L. T., "Chemical Processing and Engineering, Volume 3, Models for Flow Systems and C aemical Reactors," Marcel Dekker, New York, 1975, Chap:er 2.

22. Růžička, J., Hansen, E. H., and Mosbaek, H., Anal. Chim. Acta, 1977, 92, 235.

23. Stewart, K. K., and Rosenfeld, A. G., J. Autom. Chem., 1981, 3,30 .

24. Reijn, J. M., Van Der Linden, W. E., and Poppe, H., Anal. Chim. Acta, 1980, 114, 105.

25. Tyson, J. F., and Idris, A. B., Analyst, 1981, 106, 1125

26. Tyson, J. F., Anal. Chim. Acta, 1986, 179, 131

27. Tyson, J. F., Analyst, 1987, 112, 523.

28. Appleton, J. M. H., and Tyson, J. F., J. Anal. At. Spectrom., $1986,1,63$. 